Progress In Electromagnetics Research B, Vol. 7, 145-157, 2008

\title{
ON THE COMPLEX SYMMETRY OF THE POINCARÉ-STEKLOV OPERATOR
}

\author{
L. F. Knockaert \\ Department of Information Technology-IBBT \\ Ghent University \\ St. Pietersnieuwstraat 41, B-9000 Gent, Belgium
}

\section{De Zutter}

Department of Information Technology

Ghent University

St. Pietersnieuwstraat 41, B-9000 Gent, Belgium

\begin{abstract}
Employing Lorentz reciprocity and the Stratton-Chu formalism it is shown that the Poincaré-Steklov or admittance operator can be interpreted as a complex symmetric operator mapping the tangential electric field (instead of the equivalent magnetic current) onto the equivalent electric current. We show that the pertinent block Calderón projectors can be reformulated as operators with a block Hamiltonian structure. This leads to an explicitly complex symmetric Schur complement expression for both the interior and exterior admittance operators.
\end{abstract}

\section{INTRODUCTION}

It is well-known that the Stratton-Chu formalism [1-3] allows a complete reconstruction of the interior and exterior electromagnetic fields $(\mathbf{E}, \mathbf{H})$ inside and outside a simply connected isotropic domain $\Omega$ with smooth boundary $\partial \Omega$ merely by knowledge of the tangential field components $\left(\mathbf{E}^{t}, \mathbf{H}^{t}\right)$ [4] or the equivalent magnetic and electric currents [5] on the boundary $\partial \Omega$ in the absence of sources. A still stronger statement, resulting from the inherent duality of both Maxwell's equations and the Stratton-Chu formalism [1], is that only one tangential field component $\mathbf{E}^{t}$ or $\mathbf{H}^{t}$ (in other words only one equivalent magnetic or electric current), or even in more involved cases a non-overlapping mixture of both tangential field components 
prescribed on the boundary $\partial \Omega[6$, p. 102], is needed in order to describe the complete field configuration in the absence of source terms, except notably under resonance conditions. It follows that there exists a linear operator relationship between the equivalent magnetic and electric currents, called the Poincaré-Steklov or Dirichlet-to-Neumann operator $[4,7-10]$. In [11] it is indicated how the Poincaré-Steklov operator can be extracted from the block Calderón projectors [2] by means of a Schur complement method [12-14].

In this contribution we show, by invoking Lorentz reciprocity [15, 16], that the Poincaré-Steklov operator can be interpreted as a complex symmetric (C-symmetric) [17] operator mapping the tangential electric field (instead of the equivalent magnetic current) onto the equivalent electric current. The Poincaré-Steklov operator in the Lorentz reciprocity context is therefore a genuine admittance operator. We show that the block Calderón projectors can be reformulated as operators with a block Hamiltonian structure, well-known from Hamiltonian dynamical systems [18], Lagrangian subspace techniques [19] and algebraic Riccati equations [20]. This leads to a novel, easily understood, explicitly C-symmetric Schur complement expression for both the interior and exterior admittance operators. Finally, two analytic examples, one in connection with the magnetic eigenvector field expansion and another related to the dielectric sphere confirm the explicit C-symmetric property of the admittance operator.

\section{MAIN RESULTS}

We start with Lorentz reciprocity. Let the fields $\mathbf{E}_{1}, \mathbf{H}_{1}$ and $\mathbf{E}_{2}, \mathbf{H}_{2}$ both satisfy the source-free time-harmonic ( $e^{i \omega t}$ time dependence) Maxwell equations in the simply connected domain $\Omega \subset R^{3}$ :

$$
\begin{aligned}
\nabla \times \mathbf{E} & =-i \omega \mu \mathbf{H} \\
\nabla \times \mathbf{H} & =i \omega \epsilon \mathbf{E}
\end{aligned}
$$

It is easily proved that

$$
\nabla \cdot\left(\mathbf{E}_{1} \times \mathbf{H}_{2}-\mathbf{E}_{2} \times \mathbf{H}_{1}\right)=0
$$

implying the Lorentz reciprocity law $[15,16]$

$$
\int_{\partial \Omega}\left[\mathbf{E}_{1} \times \mathbf{H}_{2}-\mathbf{E}_{2} \times \mathbf{H}_{1}\right] \cdot \mathbf{n} d S=0
$$

where $\mathbf{n}$ is the outward pointing normal. The Lorentz reciprocity law (4) can easily be reformulated in terms of the tangential components 
$\mathbf{E}^{t}$ and $\mathbf{H}^{t}$ of the fields as

$$
\int_{\partial \Omega}\left[\mathbf{E}_{1}^{t} \times \mathbf{H}_{2}^{t}-\mathbf{E}_{2}^{t} \times \mathbf{H}_{1}^{t}\right] \cdot \mathbf{n} d S=0
$$

or

$$
\int_{\partial \Omega}\left[\mathbf{E}_{1}^{t} \cdot\left(\mathbf{n} \times \mathbf{H}_{2}^{t}\right)-\mathbf{E}_{2}^{t} \cdot\left(\mathbf{n} \times \mathbf{H}_{1}^{t}\right)\right] d S=0
$$

Since this is true for all possible tangential fields, this implies there is a complex symmetric operator (the admittance or Poincaré-Steklov operator) $\mathcal{Y}=\mathcal{Y}^{\prime}$ mapping $\mathbf{E}^{t}=-\mathbf{n} \times(\mathbf{n} \times \mathbf{E})$ onto $\mathbf{n} \times \mathbf{H}^{t}=\mathbf{n} \times \mathbf{H}$. It is important to note that the transpose operator $\mathcal{Y}^{\prime}$ is defined with respect to the 'pseudo' inner product [21, p. 113] or bilinear [17] inner product $^{\dagger}$

$$
\langle\mathbf{X} \mid \mathbf{Y}\rangle=\int_{\partial \Omega} \mathbf{X} \cdot \mathbf{Y} d S
$$

In other words, the Lorentz reciprocity relationship (6) implies that

$$
\mathbf{n} \times \mathbf{H}=\mathcal{Y}[-\mathbf{n} \times(\mathbf{n} \times \mathbf{E})]
$$

where $\mathcal{Y}$ is a $\mathrm{C}$-symmetric operator. If $\mathcal{N}$ denotes the local operator $\mathbf{n} \times$, the non-C-symmetric operator mapping the equivalent magnetic current $\mathbf{M}=\mathbf{n} \times \mathbf{E}$ onto the equivalent electric current $\mathbf{J}=\mathbf{n} \times \mathbf{H}$ $[1,5]$ is $\mathcal{S}=-\mathcal{Y N}{ }^{\ddagger}$ Although it is not C-symmetric, the operator $\mathcal{S}$ has, somewhat misleadingly, been coined admittance operator in [4] and [11]. Also, interestingly, formula (8) can simply be written as

$$
\mathbf{J}=\mathcal{Y} \mathbf{E}^{t}
$$

which has a distinctively nice reciprocal electrical network flavor: an infinity of ports on the boundary $\partial \Omega$ with 'voltages' $\mathbf{E}^{t}$ (in $\mathrm{V} / \mathrm{m}$ ) and currents $\mathbf{J}$ (in $\mathrm{A} / \mathrm{m}$ ) which are related by means of a $\mathrm{C}$-symmetric admittance operator $\mathcal{Y}$.

Next define the wavenumber, characteristic impedance and admittance as

$$
k=\omega \sqrt{\epsilon \mu} \quad z_{0}=\frac{\omega \mu}{k} \quad y_{0}=\frac{1}{z_{0}}=\frac{\omega \epsilon}{k}
$$

the scalar Green's function as

$$
g_{0}(r)=\frac{e^{-i k r}}{4 \pi r}
$$

$\dagger$ In contradistinction with the sesquilinear Hermitian inner product $[\mathbf{X} \mid \mathbf{Y}]=\left\langle\mathbf{X} \mid \mathbf{Y}^{*}\right\rangle$. Note that the bilinear inner product $\langle\mathbf{X} \mid \mathbf{Y}\rangle$ can also be interpreted as an electromagnetic 'reaction' [22].

$\ddagger$ There is no uniformity in the definitions and signs of $\mathbf{J}$ and $\mathbf{M}$ in the literature. E.g. in [1] one uses $\mathbf{M}=\mathbf{n} \times \mathbf{E}, \mathbf{J}=-\mathbf{n} \times \mathbf{H}$ and in [5] one finds $\mathbf{M}=-\mathbf{n} \times \mathbf{E}, \mathbf{J}=\mathbf{n} \times \mathbf{H}$. Here we adopt the 'default' convention $\mathbf{M}=\mathbf{n} \times \mathbf{E}$ and $\mathbf{J}=\mathbf{n} \times \mathbf{H}$. 
the operator $\mathcal{T}[2]$ operating on a tangential field $\mathbf{X}$ as

$$
\begin{aligned}
\mathcal{T} \mathbf{X} & =\mathbf{n} \times\left\{-i k \int_{\partial \Omega} g_{0}(R) \mathbf{X}(\mathbf{y}) d S_{y}+\frac{i}{k} \oint_{\partial \Omega} \nabla_{y} g_{0}(R) \nabla_{y}^{t} \cdot \mathbf{X}(\mathbf{y}) d S_{y}\right\} \\
& =-i k \mathbf{n} \times \oint_{\partial \Omega} \mathbf{G}(\mathbf{x}, \mathbf{y}) \cdot \mathbf{X}(\mathbf{y}) d S_{y}
\end{aligned}
$$

and similarly the operator $\mathcal{K}[2]$ as

$$
\begin{aligned}
\mathcal{K} \mathbf{X} & =\mathbf{n} \times \int_{\partial \Omega} \nabla_{y} g_{0}(R) \times \mathbf{X}(\mathbf{y}) d S_{y} \\
& =-\mathbf{n} \times \nabla_{x} \times \int_{\partial \Omega} \mathbf{G}(\mathbf{x}, \mathbf{y}) \cdot \mathbf{X}(\mathbf{y}) d S_{y}
\end{aligned}
$$

In the defining equations (12)-(13) $R$ is the Euclidean distance $\|\mathbf{x}-\mathbf{y}\|$ and $\oint$ denotes the Cauchy principal value integral. $\mathbf{G}(\mathbf{x}, \mathbf{y})$ is the dyadic Green's function

$$
\mathbf{G}(\mathbf{x}, \mathbf{y})=\left(\mathbf{I}+\frac{\nabla_{x} \nabla_{x}}{k^{2}}\right) g_{0}(R)
$$

Note that $\mathcal{K}$ is a compact operator $[23]^{\S}$, while $\mathcal{T}$ contains a hypersingular component necessitating the Cauchy principal value operation. Some other salient features of the operator $\mathcal{T}$ are described in Lemma 2 of [11]. The equations for the equivalent currents $\mathbf{M}=\mathbf{n} \times \mathbf{E}$ and $\mathbf{J}=\mathbf{n} \times \mathbf{H}$ are [2]

$$
\mathcal{P}\left(\begin{array}{c}
\mathbf{M} \\
z_{0} \mathbf{J}
\end{array}\right)=\left(\begin{array}{c}
\mathbf{M} \\
z_{0} \mathbf{J}
\end{array}\right)
$$

where $\mathcal{P}$ is the block Calderón operator

$$
\mathcal{P}=\left(\begin{array}{cc}
\frac{1}{2} \mathcal{I}+\mathcal{K} & -\mathcal{T} \\
\mathcal{T} & \frac{1}{2} \mathcal{I}+\mathcal{K}
\end{array}\right)
$$

The operator $\mathcal{P}$ is an idempotent (or oblique projector), i.e., $\mathcal{P}^{2}=\mathcal{P}$. This implies the well-known Calderón identities:

$$
\mathcal{T} \mathcal{K}+\mathcal{K} \mathcal{T}=0, \quad \mathcal{K}^{2}-\mathcal{T}^{2}=\frac{1}{4} \mathcal{I}
$$

Note that the identities (17) can be written in shorthand notation as

$$
(\mathcal{K} \pm i \mathcal{T})^{2}=\frac{1}{4} \mathcal{I}
$$

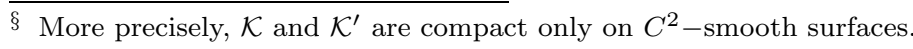


The complementary idempotent $\mathcal{I}-\mathcal{P}$, of importance for the exterior problem, is obtained by replacing $\mathcal{K}$ with $-\mathcal{K}$ and $\mathcal{T}$ with $-\mathcal{T}$. Equation (15) can be written as

$$
\left(\begin{array}{cc}
\frac{1}{2} \mathcal{I}+\mathcal{K} & -\mathcal{T} \\
\mathcal{T} & \frac{1}{2} \mathcal{I}+\mathcal{K}
\end{array}\right)\left(\begin{array}{c}
\mathcal{N} \mathbf{E}^{t} \\
z_{0} \mathbf{J}
\end{array}\right)=\left(\begin{array}{c}
\mathcal{N} \mathbf{E}^{t} \\
z_{0} \mathbf{J}
\end{array}\right)
$$

It is easily observed that $\mathcal{T}=\mathcal{N U}$, where $\mathcal{U}$ is the $\mathrm{C}$-symmetric operator $\left(\mathcal{U}=\mathcal{U}^{\prime}\right)$ defined as

$$
\mathcal{U} \mathbf{X}=-i k \int_{\partial \Omega} g_{0}(R) \mathbf{X}(\mathbf{y}) d S_{y}+\frac{i}{k} \oint_{\partial \Omega} \nabla_{y}^{t} g_{0}(R) \nabla_{y}^{t} \cdot \mathbf{X}(\mathbf{y}) d S_{y}
$$

Since $\mathcal{N}^{\prime}=-\mathcal{N}$ and $\mathcal{N}^{2}=-\mathcal{I}$, equation (19) can be reformulated as

$$
\mathcal{H}\left(\begin{array}{c}
\mathbf{E}^{t} \\
z_{0} \mathbf{J}
\end{array}\right)=\frac{1}{2}\left(\begin{array}{c}
\mathbf{E}^{t} \\
z_{0} \mathbf{J}
\end{array}\right)
$$

where

$$
\mathcal{H}=\left(\begin{array}{cc}
-\mathcal{N} & 0 \\
0 & \mathcal{I}
\end{array}\right)\left(\begin{array}{cc}
\mathcal{K} & -\mathcal{T} \\
\mathcal{T} & \mathcal{K}
\end{array}\right)\left(\begin{array}{cc}
\mathcal{N} & 0 \\
0 & \mathcal{I}
\end{array}\right)=\left(\begin{array}{cc}
-\mathcal{N} \mathcal{K N} & -\mathcal{U} \\
\mathcal{N} \mathcal{U N} & \mathcal{K}
\end{array}\right)
$$

We prove in Appendix A that $\mathcal{N} \mathcal{K N}=\mathcal{K}^{\prime}$, and hence the block operator $\mathcal{H}$ can be written as

$$
\mathcal{H}=\left(\begin{array}{cc}
-\mathcal{K}^{\prime} & -\mathcal{U} \\
\mathcal{N} \mathcal{U N} & \mathcal{K}
\end{array}\right)
$$

Now, a block operator of the form

$$
\left(\begin{array}{cc}
\mathcal{A} & \mathcal{B} \\
\mathcal{C} & -\mathcal{A}^{\prime}
\end{array}\right)
$$

is called Hamiltonian $[18,19]$ provided $\mathcal{B}$ and $\mathcal{C}$ are $\mathrm{C}$-symmetric, i.e., $\mathcal{B}^{\prime}=\mathcal{B}$ and $\mathcal{C}^{\prime}=\mathcal{C}$. Since $\mathcal{U}^{\prime}=\mathcal{U}$ and $(\mathcal{N U N})^{\prime}=\mathcal{N} \mathcal{U N}$, it is clearly seen from equation $(23)$ that $\mathcal{H}$ has a Hamiltonian structure. Moreover, utilizing the Calderón identities (17) it is easily proved that

$$
\mathcal{H}^{2}=\frac{1}{4} \mathcal{I}
$$

Inserting $\mathbf{J}=\mathcal{Y} \mathbf{E}^{t}$ in formula (21), it is seen that the admittance operator $\mathcal{Y}$ satisfies the two operator equations

$$
\begin{aligned}
-\mathcal{K}^{\prime}-z_{0} \mathcal{U Y} & =\frac{1}{2} \mathcal{I} \\
\mathcal{N U N}+z_{0} \mathcal{K Y} & =\frac{1}{2} z_{0} \mathcal{Y}
\end{aligned}
$$


Solving (26) for $z_{0} \mathcal{Y}$ yields

$$
z_{0} \mathcal{Y}=-\mathcal{U}^{-1}\left(\frac{1}{2} \mathcal{I}+\mathcal{K}^{\prime}\right)
$$

provided the operator $\mathcal{U}$ is invertible, which we assume (see also $[11,10])$. Rewriting operator equation $(27)$ as

$$
z_{0} \mathcal{Y}=\mathcal{N} \mathcal{U N}+z_{0}\left(\frac{1}{2} \mathcal{I}+\mathcal{K}\right) \mathcal{Y}
$$

and inserting the solution (28) in the r.h.s. of (29) results in the explicitly C-symmetric Schur complement form of the admittance operator

$$
\mathcal{Y}=y_{0}\left[\mathcal{N} \mathcal{U N}-\left(\frac{1}{2} \mathcal{I}+\mathcal{K}\right) \mathcal{U}^{-1}\left(\frac{1}{2} \mathcal{I}+\mathcal{K}^{\prime}\right)\right]
$$

Similarly, the exterior problem with Calderón projector $\mathcal{I}-\mathcal{P}$ and Hamiltonian $-\mathcal{H}$ results in the explicitly C-symmetric Schur complement form of the exterior admittance operator

$$
\tilde{\mathcal{Y}}=-y_{0}\left[\mathcal{N} \mathcal{U N}-\left(\frac{1}{2} \mathcal{I}-\mathcal{K}\right) \mathcal{U}^{-1}\left(\frac{1}{2} \mathcal{I}-\mathcal{K}^{\prime}\right)\right]
$$

It is also easily proved that both $\mathcal{Y}$ and $\tilde{\mathcal{Y}}$ are C-symmetric solutions of the operator Riccati equation

$$
\mathcal{N U N}+z_{0} \mathcal{K Y}+z_{0} \mathcal{Y} \mathcal{K}^{\prime}+z_{0}^{2} \mathcal{Y U Y}=0
$$

\subsection{Remark: Galerkin Approximation}

The formula (21) forms an excellent starting point to obtain a projected Galerkin approximation for the admittance operator with preservation of symmetry. The pertinent equations are:

$$
\begin{aligned}
-\mathcal{K}^{\prime} \mathbf{E}^{t}-z_{0} \mathcal{U} \mathbf{J} & =\frac{1}{2} \mathbf{E}^{t} \\
\mathcal{N U \mathcal { N }} \mathbf{E}^{t}+z_{0} \mathcal{K} \mathbf{J} & =\frac{1}{2} z_{0} \mathbf{J} \\
\mathbf{J} & =\mathcal{Y} \mathbf{E}^{t}
\end{aligned}
$$

We expand $\mathbf{E}^{t}$ and $\mathbf{J}$ as

$$
\begin{aligned}
\mathbf{E}^{t} & =\sum_{l=1}^{N} \alpha_{l} \mathbf{b}_{l}(\mathbf{x}) \\
\mathbf{J} & =\sum_{l=1}^{N} \beta_{l} \mathbf{b}_{l}(\mathbf{x})
\end{aligned}
$$


where the basis functions $\left\{\mathbf{b}_{l}(\mathbf{x})\right\}$ are supposed real-valued. Inserting the expansions (36)-(37) in the formulas (33)-(35) and testing the resulting equations with the same set of basis functions yields the system of linear equations

$$
\begin{aligned}
-\mathrm{A}^{T} \alpha-z_{0} \mathrm{~B} \beta & =\frac{1}{2} \mathrm{D} \alpha \\
\mathrm{C} \alpha+z_{0} \mathrm{~A} \beta & =\frac{1}{2} z_{0} \mathrm{D} \beta \\
\mathrm{D} \beta & =\mathrm{Y} \alpha
\end{aligned}
$$

where the $N \times N$ matrices $\mathrm{A}, \mathrm{B}, \mathrm{C}, \mathrm{D}, \mathrm{Y}$ are defined as

$$
\begin{aligned}
& \mathrm{A}_{k, l}=\left\langle\mathbf{b}_{k} \mid \mathcal{K} \mathbf{b}_{l}\right\rangle \quad \mathrm{B}_{k, l}=\left\langle\mathbf{b}_{k} \mid \mathcal{U} \mathbf{b}_{l}\right\rangle \quad \mathrm{D}_{k, l}=\left\langle\mathbf{b}_{k} \mid \mathbf{b}_{l}\right\rangle \\
& \mathrm{C}_{k, l}=\left\langle\mathbf{b}_{k} \mid \mathcal{N} \mathcal{U} \mathcal{N} \mathbf{b}_{l}\right\rangle \quad \mathrm{Y}_{k, l}=\left\langle\mathbf{b}_{k} \mid \mathcal{Y} \mathbf{b}_{l}\right\rangle
\end{aligned}
$$

The matrices $B, C$ and $D$ are all complex symmetric, and it easily shown that the Galerkin projected admittance matrix $Y$ can be written in the explicitly complex symmetric Schur complement form as

$$
\mathrm{Y}=y_{0}\left[\mathrm{C}-\left(\frac{1}{2} \mathrm{D}+\mathrm{A}\right) \mathrm{B}^{-1}\left(\frac{1}{2} \mathrm{D}+\mathrm{A}^{T}\right)\right]
$$

which has a similar explicitly complex symmetric Schur structure as the operator equation (30).

\section{EXAMPLES}

In this section we give two validating examples of the C-symmetry property of the admittance operator. The first example involves the general description of the interior admittance operator in terms of the magnetic eigenvectors for the domain $\Omega$ and the second example derives the admittance operator of a dielectric sphere in terms of vector spherical harmonics.

\subsection{Magnetic Eigenvector Expansion}

Consider the expansion of the magnetic field in terms of the magnetic eigenvectors pertaining to the domain $\Omega$. It has been shown in $[15$, p. 299] and [16, p. 516] that the magnetic field inside $\Omega$ can be written in terms of the tangential electric field as

$$
\begin{aligned}
\mathbf{H}(\mathbf{x})= & -\frac{1}{i \omega \mu} \sum_{m} \mathbf{g}_{m}(\mathbf{x}) \int_{\partial \Omega}\left(\mathbf{n}_{y} \times \mathbf{E}^{t}(\mathbf{y})\right) \cdot \mathbf{g}_{m}(\mathbf{y}) d S_{y} \\
& +\sum_{m} \frac{i \omega \epsilon}{k^{2}-k_{m}^{2}} \mathbf{h}_{m}(\mathbf{x}) \int_{\partial \Omega}\left(\mathbf{n}_{y} \times \mathbf{E}^{t}(\mathbf{y})\right) \cdot \mathbf{h}_{m}(\mathbf{y}) d S_{y}
\end{aligned}
$$


where $\mathbf{g}_{m}(\mathbf{x})$ and $\mathbf{h}_{m}(\mathbf{x})$ are respectively the orthonormalized irrotational and solenoidal magnetic eigenvectors [16] pertaining to $\Omega$ and the $k_{m}^{2}$ are the solenoidal magnetic eigenvalues. Defining the rotated eigenvectors over $\partial \Omega$ as

$$
\tilde{\mathbf{g}}_{m}(\mathbf{x})=\mathbf{n}_{x} \times \mathbf{g}_{m}(\mathbf{x}) \quad \tilde{\mathbf{h}}_{m}(\mathbf{x})=\mathbf{n}_{x} \times \mathbf{h}_{m}(\mathbf{x})
$$

it is easily seen that we can write

$$
\begin{aligned}
\mathbf{J}(\mathbf{x})= & \frac{1}{i \omega \mu} \sum_{m} \tilde{\mathbf{g}}_{m}(\mathbf{x}) \int_{\partial \Omega} \tilde{\mathbf{g}}_{m}(\mathbf{y}) \cdot \mathbf{E}^{t}(\mathbf{y}) d S_{y} \\
& +\sum_{m} \frac{i \omega \epsilon}{k_{m}^{2}-k^{2}} \tilde{\mathbf{h}}_{m}(\mathbf{x}) \int_{\partial \Omega} \tilde{\mathbf{h}}_{m}(\mathbf{y}) \cdot \mathbf{E}^{t}(\mathbf{y}) d S_{y}
\end{aligned}
$$

Defining the matrix kernel of $\mathcal{Y}$ as $\mathrm{Y}(\mathbf{x}, \mathbf{y})$, we find that

$$
\mathbf{Y}(\mathbf{x}, \mathbf{y})=\frac{1}{i \omega \mu} \sum_{m} \tilde{\mathbf{g}}_{m}(\mathbf{x}) \otimes \tilde{\mathbf{g}}_{m}(\mathbf{y})+\sum_{m} \frac{i \omega \epsilon}{k_{m}^{2}-k^{2}} \tilde{\mathbf{h}}_{m}(\mathbf{x}) \otimes \tilde{\mathbf{h}}_{m}(\mathbf{y})
$$

where $\otimes$ is the dyadic or outer product. It is clear that $\mathrm{Y}(\mathbf{x}, \mathbf{y})=$ $\mathrm{Y}^{T}(\mathbf{y}, \mathbf{x})$ ( $T$ being the matrix transpose), implying that $\mathcal{Y}$ is $\mathrm{C}$ symmetric.

\subsection{The Dielectric Sphere}

We start with the general vector spherical harmonics decomposition of the electromagnetic fields [24, p. 444]:

$$
\begin{aligned}
\mathbf{E}(\mathbf{x}) & =\sum_{n=0}^{\infty} \sum_{m=-n}^{n}\left[a_{m n} \mathbf{q}_{m n}(\mathbf{x})+b_{m n} \mathbf{p}_{m n}(\mathbf{x})\right] \\
\mathbf{H}(\mathbf{x}) & =i y_{0} \sum_{n=0}^{\infty} \sum_{m=-n}^{n}\left[a_{m n} \mathbf{p}_{m n}(\mathbf{x})+b_{m n} \mathbf{q}_{m n}(\mathbf{x})\right]
\end{aligned}
$$

where the vector spherical harmonics in spherical coordinates are:

$$
\mathbf{p}_{m n}(\mathbf{x})=\left[\frac{i m}{\sin \theta} \mathrm{P}_{n}^{|m|}(\cos \theta) \mathbf{u}_{\theta}-\frac{d}{d \theta} \mathrm{P}_{n}^{|m|}(\cos \theta) \mathbf{u}_{\phi}\right] j_{n}(k r) e^{i m \phi}
$$

and

$$
\begin{aligned}
\mathbf{q}_{m n}(\mathbf{x})= & {\left[\frac{n(n+1)}{k r} j_{n}(k r) \mathrm{P}_{n}^{|m|}(\cos \theta) \mathbf{u}_{r}+\frac{1}{k r} \frac{d}{d r}\left\{r j_{n}(k r)\right\} \frac{d}{d \theta} \mathrm{P}_{n}^{|m|}(\cos \theta) \mathbf{u}_{\theta}\right.} \\
& \left.+\frac{i m}{k r \sin \theta} \frac{d}{d r}\left\{r j_{n}(k r)\right\} \mathrm{P}_{n}^{|m|}(\cos \theta) \mathbf{u}_{\phi}\right] e^{i m \phi}
\end{aligned}
$$


The tangential fields are

$$
\begin{aligned}
\mathbf{E}^{t}(\mathbf{x}) & =\sum_{n=0}^{\infty} \sum_{m=-n}^{n}\left[a_{m n} \mathbf{q}_{m n}^{t}(\mathbf{x})+b_{m n} \mathbf{p}_{m n}(\mathbf{x})\right] \\
\mathbf{H}^{t}(\mathbf{x}) & =i y_{0} \sum_{n=0}^{\infty} \sum_{m=-n}^{n}\left[a_{m n} \mathbf{p}_{m n}(\mathbf{x})+b_{m n} \mathbf{q}_{m n}^{t}(\mathbf{x})\right]
\end{aligned}
$$

where

$$
\mathbf{q}_{m n}^{t}(\mathbf{x})=\left[\frac{d}{d \theta} \mathrm{P}_{n}^{|m|}(\cos \theta) \mathbf{u}_{\theta}+\frac{i m}{\sin \theta} \mathrm{P}_{n}^{|m|}(\cos \theta) \mathbf{u}_{\phi}\right] \frac{1}{k r} \frac{d}{d r}\left\{r j_{n}(k r)\right\} e^{i m \phi}
$$

It is straightforward to show that

$$
\begin{aligned}
\mathbf{u}_{r} \times \mathbf{q}_{m n}^{t} & =-\alpha_{n} \mathbf{p}_{m n} \\
\mathbf{u}_{r} \times \mathbf{p}_{m n} & =\frac{1}{\alpha_{n}} \mathbf{q}_{m n}^{t}
\end{aligned}
$$

where

$$
\alpha_{n}=\frac{\frac{d}{d r}\left\{r j_{n}(k r)\right\}}{k r j_{n}(k r)}
$$

Hence $\mathbf{J}(\mathbf{x})=\mathbf{u}_{r} \times \mathbf{H}^{t}(\mathbf{x})$ can be written as

$$
\mathbf{J}(\mathbf{x})=i y_{0} \sum_{n=0}^{\infty} \sum_{m=-n}^{n}\left[\frac{a_{m n}}{\alpha_{n}} \mathbf{q}_{m n}^{t}(\mathbf{x})-\alpha_{n} b_{m n} \mathbf{p}_{m n}(\mathbf{x})\right]
$$

Due to the solid angle orthogonality relations $(d \sigma=\sin \theta d \theta d \phi)$

$$
\begin{aligned}
\int \mathbf{p}_{m n} \cdot \mathbf{p}_{-r, s} d \sigma & =\delta_{m r} \delta_{n s} P_{m n} \\
\int \mathbf{q}_{m n}^{t} \cdot \mathbf{q}_{-r, s}^{t} d \sigma & =\delta_{m r} \delta_{n s} Q_{m n} \\
\int \mathbf{p}_{m n} \cdot \mathbf{q}_{r, s}^{t} d \sigma & =0
\end{aligned}
$$

where

$$
\begin{aligned}
P_{m n} & =\frac{(n+|m|) !}{(n-|m|) !} \frac{4 \pi n(n+1)}{2 n+1}\left[j_{n}(k r)\right]^{2} \\
Q_{m n} & =\frac{(n+|m|) !}{(n-|m|) !} \frac{4 \pi n(n+1)}{2 n+1}\left[\frac{1}{k r} \frac{d}{d r}\left\{r j_{n}(k r)\right\}\right]^{2}
\end{aligned}
$$


we find the coefficients $a_{m, n}$ and $b_{m, n}$ as

$$
\begin{aligned}
a_{m n} & =\frac{1}{Q_{m n}} \int \mathbf{E}^{t} \cdot \mathbf{q}_{-m, n}^{t} d \sigma \\
b_{m n} & =\frac{1}{P_{m n}} \int \mathbf{E}^{t} \cdot \mathbf{p}_{-m, n} d \sigma
\end{aligned}
$$

Therefore it follows from equation (58) that the matrix kernel of the admittance operator $\mathcal{Y}$ for a sphere of radius $r$ can be written as

$$
\begin{aligned}
\mathrm{Y}(\mathbf{x}, \mathbf{y})=i y_{0} \sum_{n=0}^{\infty} \sum_{m=-n}^{n} & {\left[\frac{1}{\alpha_{n} Q_{m n}} \mathbf{q}_{m n}^{t}(\mathbf{x}) \otimes \mathbf{q}_{-m, n}^{t}(\mathbf{y})\right.} \\
& \left.-\frac{\alpha_{n}}{P_{m n}} \mathbf{p}_{m n}(\mathbf{x}) \otimes \mathbf{p}_{-m, n}(\mathbf{y})\right]
\end{aligned}
$$

Due to the fact that the parameters $P_{m n}$ and $Q_{m n}$ are even in $m$, and since the sum in (66) extends over both positive and negative $m$, it is easily shown that $\mathrm{Y}(\mathbf{x}, \mathbf{y})=\mathrm{Y}^{T}(\mathbf{y}, \mathbf{x})$, implying that $\mathcal{Y}$ is $\mathrm{C}$-symmetric. Note that the exterior admittance operator can easily be obtained by replacing the spherical Bessel functions $j_{n}(\cdot)$ with the spherical Hankel functions $h_{n}^{(2)}(\cdot)$ in the formulas where they appear.

\section{CONCLUDING REMARKS}

Employing Lorentz reciprocity and the Stratton-Chu formalism it has been shown that the admittance operator is a C-symmetric operator mapping the tangential electric field onto the equivalent electric current. This is a consequence of the fact that the block Calderón projectors can be reformulated as operators with a block Hamiltonian structure, resulting in C-symmetric Schur complement expressions for both the interior and exterior admittance operators. As a final remark, we will briefly discuss the domain and range of the operator $\mathcal{Y}$. It has been shown in [11] that the operator $\mathcal{S}=-\mathcal{Y} \mathcal{N}$, mapping the equivalent magnetic current onto the equivalent electric current, has $\operatorname{dom}(\mathcal{S})=\operatorname{ran}(\mathcal{S})=H_{\operatorname{div}}^{-1 / 2}(\partial \Omega)$. Hence the admittance operator $\mathcal{Y}$ itself has domain $\operatorname{dom}(\mathcal{Y})=H_{\text {curl }}^{-1 / 2}(\partial \Omega)$ and range $\operatorname{ran}(\mathcal{Y})=$ $H_{\text {div }}^{-1 / 2}(\partial \Omega)$. This implies that the Galerkin expansion functions $\mathbf{b}_{k}(\mathbf{x})$ must reside in

$$
H_{\text {div }, \operatorname{curl}}^{-1 / 2}(\partial \Omega)=H_{\operatorname{div}}^{-1 / 2}(\partial \Omega) \cap H_{\text {curl }}^{-1 / 2}(\partial \Omega)
$$


which is the fundamental function space [2] for tangential components of electromagnetic fields on smooth boundary surfaces $\partial \Omega$. However, notwithstanding the theoretical importance of the function space $H_{\text {div, curl }}^{-1 / 2}(\partial \Omega)$, it should be noted that the basis functions most widely used in practice are simply the well-known surface edge elements [5] and/or rotated surface edge elements [10].

\section{APPENDIX A. PROOF THAT $\mathcal{N} \mathcal{K} \mathcal{N}=\mathcal{K}^{\prime}$}

Defining the dyadic (or matrix) kernel of $\mathcal{K}$ as $\mathbf{K}(\mathbf{x}, \mathbf{y})$, it is easily understood that the kernel of $\mathcal{K}^{\prime}$ needs to be $\mathbf{K}^{T}(\mathbf{y}, \mathbf{x})$. Hence, since

$$
\mathbf{K}(\mathbf{x}, \mathbf{y})=\mathbf{n}_{x} \times \nabla_{y} g_{0}(R) \times
$$

we obtain

$$
\begin{aligned}
\mathbf{K}^{T}(\mathbf{y}, \mathbf{x}) & =\nabla_{x} g_{0}(R) \times \mathbf{n}_{y} \times \\
& =-\nabla_{y} g_{0}(R) \times \mathbf{n}_{y} \times \\
& =\left(\mathbf{n}_{x} \times\right)^{2} \nabla_{y} g_{0}(R) \times \mathbf{n}_{y} \times \\
& =\mathbf{n}_{x} \times\left(\mathbf{n}_{x} \times \nabla_{y} g_{0}(R) \times\right) \mathbf{n}_{y} \times
\end{aligned}
$$

as required. This result indirectly implies that the spectrum of the operator $\mathcal{K}$ is symmetric with respect to the origin (see also to that effect the Appendix of [2]).

\section{REFERENCES}

1. Stratton, J. A. and L. J. Chu, "Diffraction theory of electromagnetic waves," Phys. Rev., Vol. 56, No. 1, 99-107, 1939.

2. Hsiao, G. C. and R. E. Kleinman, "Mathematical foundations for error estimation in numerical solutions of integral equations in electromagnetics," IEEE Trans. Antennas Propagat., Vol. 45, No. 3, 316-328, 1997.

3. Tai, C. T., "Direct integration of field equations," Progress In Electromagnetics Research, Vol. 28, 339-359, 2000.

4. Borel, S., D. P. Levadoux, and F. Alouges, "A new wellconditioned integral formulation for Maxwell equations in three dimensions," IEEE Trans. Antennas Propagat., Vol. 53, No. 9, 2995-3004, 2005.

5. Sheng, X. Q., J. M. Jin, J. Song, W. C. Chew, and C. C. Lu, "Solution of combined-field integral equation using multilevel fast multipole algorithm for scattering by homogeneous bodies," IEEE Trans. Antennas Propagat., Vol. 46, No. 11, 1718-1726, 1998. 
6. Harrington, R. F., Time-Harmonic Electromagnetic Fields, Wiley, New York, 2001.

7. Sauter, S. and C. Schwab, Randelementmethoden, BG Teubner, Stuttgart, 2004.

8. Steinbach, O., Numerische Näherungsverfahren für elliptische Randwertprobleme, Advances in Numerical Mathematics, BG Teubner, Stuttgart, 2003.

9. Hiptmair, R., "Coupling of finite elements and boundary elements in electromagnetic scattering," SIAM J. Numer. Anal., Vol. 41, 919-944, 2003.

10. Buffa, A. and R. Hiptmair, "Galerkin boundary element methods for electromagnetic scattering," Topics in Computational Wave Propagation. Direct and Inverse Problems, M. Ainsworth, P. Davis, D. Duncan, P. Martin, B. Rynne (eds.), Vol. 31, 83124; Lecture Notes in Computational Science and Engineering Springer, Berlin, 2003.

11. de La Bourdonnaye, A., "Some formulations coupling finite element and integral equation methods for Helmholtz equation and electromagnetism," Numer. Math., Vol. 69, No. 3, 257-268, 1995.

12. Knockaert, L., D. De Zutter, G. Lippens, and H. Rogier, "On the Schur complement form of the Dirichlet-to-Neumann operator," Wave Motion, Vol. 45, No. 3, 309-324, 2008.

13. Crabtree, D. E. and E. V. Haynsworth, "An identity for the Schur complement of a matrix," Proc. Amer. Math. Soc., Vol. 22, 364366, 1969.

14. Corach, G., A. Maestripieri, and D. Stojanoff, "Generalized Schur complements and oblique projections," Lin. Alg. Appl., Vol. 341, 259-272, 2002.

15. Van Bladel, J., Electromagnetic Fields, McGraw-Hill, New York, 1964 (revised printing, Washington DC, Hemisphere, 1985).

16. Van Bladel, J. G., Electromagnetic Fields, 2nd edition, IEEE Press, Piscataway, 2007.

17. Garcia, S. R. and M. Putinar, "Complex symmetric operators and applications," Trans. Amer. Math. Soc., Vol. 358, No. 3, 12851315, 2005.

18. Meyer, K. R. and G. R. Hall, Introduction to Hamiltonian Dynamical Systems and the N-body Problem. Applied Mathematical Sciences, Vol. 90, Springer, New York, 1992.

19. Lin, W. W. and C. S. Wang, "On computing stable Lagrangian subspaces of Hamiltonian matrices and symplectic pencils," SIAM 
J. Matrix Anal. Appl., Vol. 18, 590-614, 1997.

20. Laub, A., "A Schur method for solving algebraic Riccati equations," IEEE Trans. Automat. Control, Vol. 24, 913-921, 1979.

21. Hanson, G. W. and A. B. Yakovlev, Operator Theory for Electromagnetics. An Introduction, Springer, New York, 2002.

22. Deschamps, G. A., "Electromagnetics and differential forms," Proc. IEEE, Vol. 69, No. 6, 676-696, 1981.

23. Colton, D. and R. Kress, Integral Equation Methods in Scattering Theory, Wiley, New York, 1984.

24. Jones, D. S., Acoustic and Electromagnetic Waves, Clarendon Press, Oxford, 1986. 